\title{
OPTIMIZED ORGANIZATION AND ADAPTIVE VISUALIZATION OF COMPLICATED MOUNTAIN DISASTER 3D SCENES FOR DIVERSE TERMINALS
}

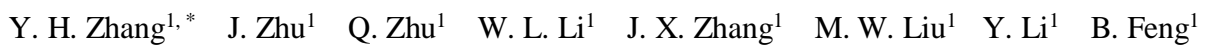 \\ ${ }^{1}$ Faculty of Geosciences and Environmental Engineering, Southwest Jiaotong University, 611756 Chengdu, China - \\ zhangyh0506@163.com,vgezj@163.com, zhuq66@263.net, vgewilliam@163.com,412086140@qq.com, \\ liumingwei@my.swjtu.edu.cn, liyun20151202@outlook.com, 280930320@qq.com
}

KEY WORDS: Mountain Disaster; 3D Scenes; Adaptive Visualization; Optimized Organization; Diverse Terminals

\begin{abstract}
:
Mountain disaster scenes usually contains various geographical entities, which are dynamic and complicated. Therefore, the construction of mountain disaster 3D scenes has great significance for disaster simulation, analysis and prediction. 3D visualization and interactive analysis of complicated mountain disaster scenes should accommodate users who have access to various terminals in disaster emergency response. However, most of the existing 3D visualization methods can only deal with 3D scene data organization and scheduling for single terminal or limited kinds of geographical entities. Due to performance constraints, it's difficult for Mobile devices to support efficient visualization of complicated mountain disaster 3D scenes, either. To address these issues, we research the key technologies for efficient visualization of mountain disasters on diverse terminals. We utilize the B/S architecture and research its impact on rendering frame rate and the relationship of terminal characteristics and parameters through analysis of terminal characteristics and parameters, e.g. hardware performance, screen size and resolution, network environment, and rendering frame rate requirements. Then we analyze the diverse organization of mountain disaster scene data and explore the methods of constructing efficient spatial index by taking into account the characteristics of diverse terminals. An adaptive scene analysis method is subsequently designed to select the optimal model. Finally, based on the diverse organization of various data in the scene, a corresponding dynamic scheduling method is proposed to realize the adaptive visualization of complicated mountain disaster 3D scenes for diverse terminals.
\end{abstract}

\section{INTRODUCTION}

China is a mountain disaster-prone country, the mountain disaster environment usually contains various geographical entities, which are dynamic and complicated, so how to simulate and analyse the environments, the evolution and the development processes of mountain disasters is a key scientific problem which needs to be solved urgently (Cui. 2014). Construction of disaster virtual environment could provide a new method for disaster prediction and analysis (Denolle et al. 2014). Therefore, it is of great significance to construct virtual geographic environment to assist the simulation, analysis, prediction and decision -making in disasters (Lin et al. 2013; Chen et al. 2015; Kim et al. 2016; Lin et al. 2016).

Because the time and location of the disaster is uncertain, the locations of people who have access to various terminals in disaster emergency response are distributed differently, and the disaster visualization not only needs to deal with various objects in different fields, levels and complicated structures, but also to meet the diverse visualization requirements of different user preferences, information requirements and terminal constraints. Therefore, 3D visualization and interactive analysis of complicated mountain disaster scenes for diverse terminals is a trend. Because of the dynamic complexity of the disaster scenes, and the different types of disaster areas need to focus on the difference of the scene objects, there is not a complete and universal visualization method at present, which can not only describe the features of all the scene objects accurately, but also meet the requirements of all the users in the field. In the current researches of disaster scenes, most of these researches focus on a single disaster scene, and the terminal type of visualization and analysis are single, it's hard to meet the requirements of the complicated scene effective visualization.

To address these issues, we research the key technologies for efficient and visualization of complicated mountain disasters on diverse terminals. We utilize the $\mathrm{B} / \mathrm{S}$ architecture and analyze the effects of terminal performance, screen size, screen resolution, network environment, viewpoint information and interactive mode of diverse terminals on disaster scene rendering efficiency. According to research the characteristics of disaster scene data, which contain image and terrain data, 3D building data, disaster simulation data and so on, we explore the methods of optimized organization and constructing efficient spatial index by taking into account the characteristics of diverse terminals and propose the adaptive scheduling method considering disaster scene rendering efficiency and visualization quality (Zhang et al. 2017). Finally, we would choose the flood scene as typical mountain disaster to construct a disaster 3D scene and select three types of terminals for experiments to the effectiveness of the proposed method.

\section{DESIGN OF ADAPTIVE FRAMEWORK FOR DIVERSE TERMINALS}

Mountain disaster scenes usually contain a large amount of data, include image and terrain data, 3D building models, disaster simulation data, POI data and road models (Hu et al. 2018). Therefore, the optimization organization and adaptive scheduling of disaster scene data is the key point to the high efficiency and high quality $3 \mathrm{D}$ visualization of complicated disaster scenes for diverse terminals. So we utilize the B/S architecture for adaptive visualization, as shown in Figure 1. 


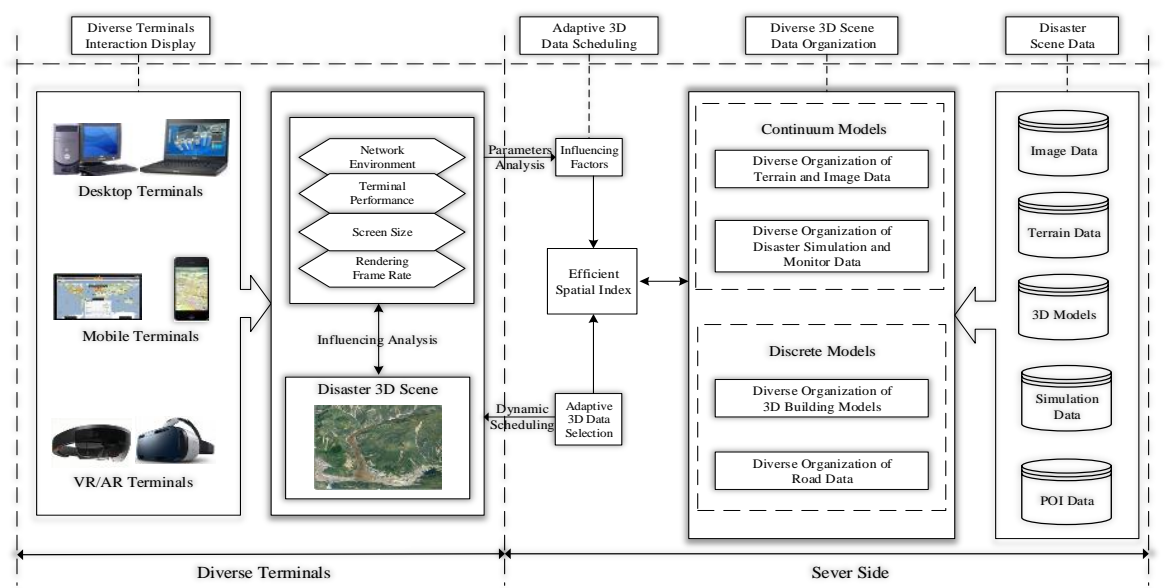

Figure 1. Adaptive Visualization of Complicated Disaster Scenes Based on B/S Architecture for Diverse Terminals

On the server side, we research the characteristics some typical kinds of disaster data, include image and terrain data, 3D building data, disaster simulation data and POI Data, and complete the diverse organization of these disaster data. The organization method of original disaster data is to process the 3D data into multi-level LOD, and construct multi-resolution scene pyramid. Aiming at diverse terminals and network environment, and complete the optimal selection for various data of disaster scene by constructing different resolution pyramid structures and selecting different LOD levels of data. In chapter 3, we would explain the organization and scheduling method of different disaster data.

On the browser side, the network environment, terminal performance and screen size are identified, and real-time rendering frame rate is monitored. Then we complete the adaptive LODs hierarchy selection and dynamic scheduling, and the fusion modeling method of various disaster 3D scenes and the construction and visualization of complicated mountain disaster scenes based on WebGL.

\section{THE CONSTRUCTION AND OPTIMIZATION OF DISASTER 3D SCENES}

Compared with the desktop terminals, the mobile terminals have poor performance and small screen size. In order to adapt to the efficient 3D visualization for mobile terminals, we need to optimize the organization of disaster scene data to reduce the rendering data size on the browser side and improve the efficiency of data transmission and rendering. We optimize organization and dynamic scheduling for different disaster data to meet the requirements of adaptive and efficient visualization on diverse terminals.

\subsection{Diverse Organization of Terrain and Image Data}

For image and terrain data, we propose two methods which is controlling the maximum tile level and the size of the loaded tile to reduce the rendering data size on the browser side to improve the efficiency of visualization. For example, the desktop terminals load the $512 * 512$ resolution of maximum L4 LOD level tiles, the mobile terminals load the $256 * 256$ resolution of maximum L3 LOD level tiles, as shown in Figure 2 (Hu et al. 2018).
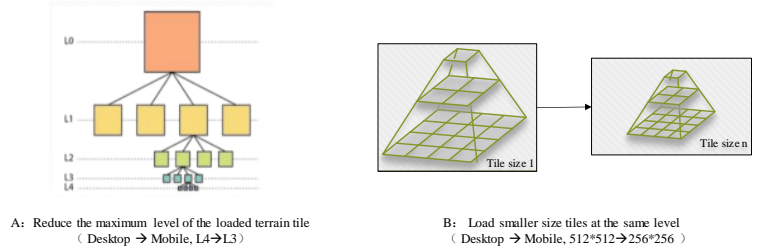

Figure 2. The Method of Controlling the Maximum Tile Level and Size of Loaded Tile

\subsection{Optimized Organization and Scheduling of 3D Building Models}

For 3D building models, we processed the $3 \mathrm{D}$ data into multilevel LOD. The detailed LOD $n+1$ model was hierarchically organized as LOD $0 \sim$ LOD $n$ hierarchical multi-texture resolution model, LOD 0 model was the blank texture and geometric simplification model. The texture of each LOD level of the model was reduced by $2 \times 2$ resolution, these LOD building models as shown in Figure 3. As the performance of terminals was decreased, the client could select lower texture resolution 3D model. As the performance of terminals was decreased, the client would select lower texture resolution 3D building model.

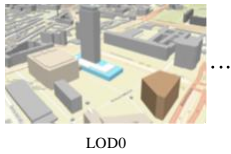

Figure 3. Multi-level LOD Building Models

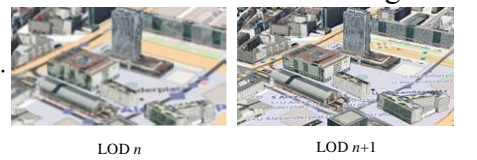

The 3D building models in complicated mountain disaster scene usually have the characteristics of large scale, uneven distribution, and large density. The traditional quad-tree organization method does not consider the distribution characteristics of models in the spatial region, and the traditional organization constructs a static spatial index for a single terminal, it is difficult to adapt to the requirements of visualization for diversified terminals (Chen et al. 2017).

Therefore, we proposed adaptive quad-tree division, this method ensures the balance of the number of 3D models in each division region, avoids the $3 \mathrm{D}$ model at the edge of the $3 \mathrm{D}$ scene to be divided into different regions, maintains better topology and semantic information, supports the smooth transition of the multiple level of detail model, and maintains better global and local details of buildings, improve scene authenticity, and enhance visual rendering effect. And we designed dynamic spatial index, the attributes of index as shown in Table 1 (Zhang et al. 2017). In the construction process of dynamic spatial index, 
the initial setting of the geometric error values is determined by terminal performance, screen size and network environment. In the process of visualization, the real-time rendering frame rate is compared with the initial setting rendering frame rate thresholds. If it is lower than the setting threshold, the geometric error value is dynamically modified to adjust the amount of data in real-time until the real-time rendering frame rate is higher than thresholds. The adaptive quad-tree organization method as shown in Figure 4.

\begin{tabular}{|c|c|}
\hline Attributes & Attributes Instructions \\
\hline BoundingVolume & Defined Bounding Volume \\
\hline GeometricError & $\begin{array}{c}\text { Defined the Geometric Error } \\
\text { When Rendering Current Node }\end{array}$ \\
\hline Children & Defined Children Nodes Collections \\
\hline Content & Define the Current Node Contents \\
\hline
\end{tabular}

Table 1. Attributes of Spatial Index

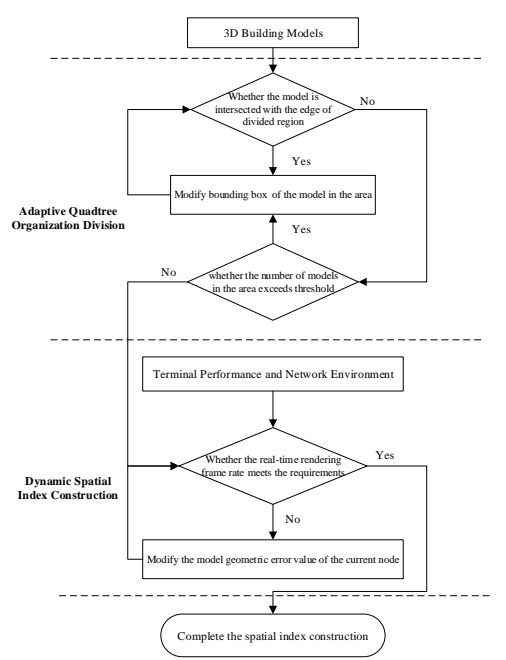

Figure 4. Adaptive Quad-tree Organization Method

\subsection{Disaster Simulation Data Scenes Optimization}

We select three typical mountain disasters to research disaster simulation data optimization, flood, debris flow and landslide. For flood and debris flow simulation data, we all construct the disaster simulation data scene through construction of triangulation network. We take flood data as an example, the flood simulation data consist of a series of grids with attribute values for water depth, elevation and flow velocity. Grids with water cells are used to build a triangulation network, which can reduce the amount of data and accurately represent the flood surface. To improve the efficiency of data transmission and rendering, the above flood data can be simplified in different LOD models to reduce the data size (Liu et al. 2017; Yin et al. 2017). As the performance of terminals was decreased, the client would select the lower LOD level flood data. The flood scene optimization method as shown in Figure 5, the flood simulation visualization is shown in Figure 6, the debris flow simulation visualization is shown in Figure 7.

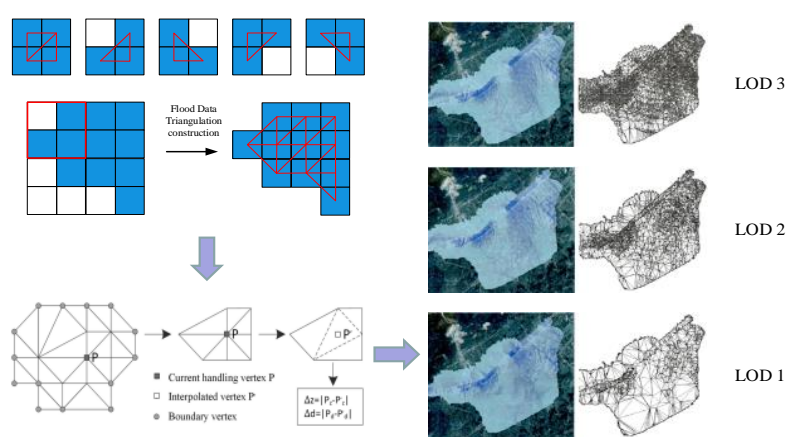

Figure 5. Flood Scene Optimization Method

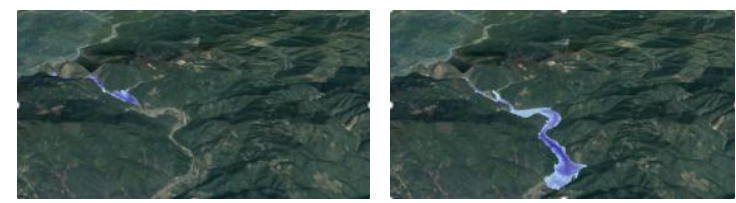

Figure 6. Flood Simulation Visualization
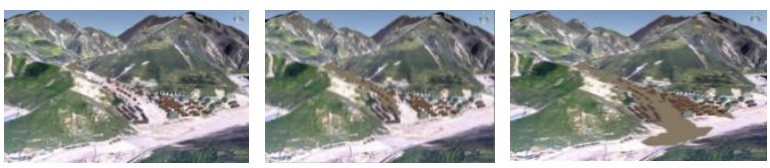

Figure 7. Debris Flow Simulation Visualization

For landslide simulation data, we extract the landslide boundary, body and accumulation body based on imagery and terrain data. Then the landslide simulation results are interpolated to generated the spatial-temporal data and get DEM in the process of landslides at different times based on the mechanism of landslide movement, and we use particles to express the divided landslide area as shown in Figure 8, the partition granularity of DEM is determined by terminal type, the partition granularity is smaller, the more particles will be used to express the landslide, the better the visualization effect. Finally, we use realistic texture mapping to enhance the reality of landslide scene and use GPU to accelerate rendering to complete the efficient visualization of landslide. The landslide simulation data optimization method is shown as Figure 9, the landslide simulation visualization is shown in Figure 10.
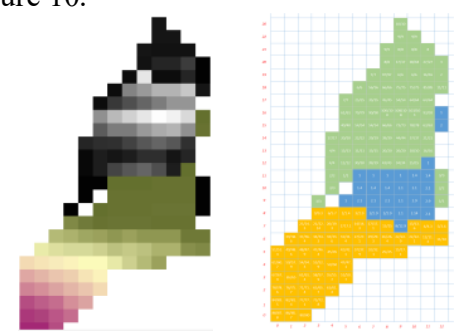

Figure 8. The Particles to Express the Divided Landslide Area

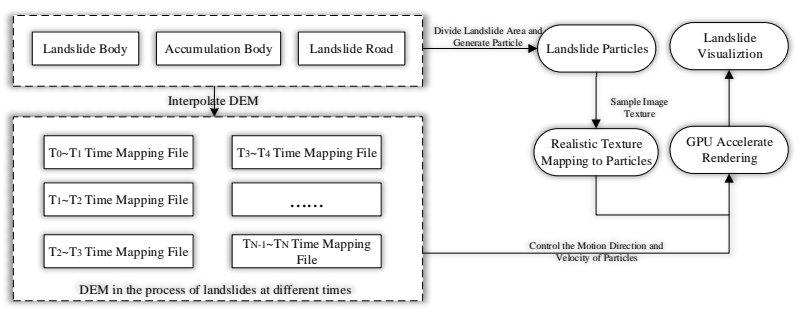

Figure 9. The Landslide Simulation Data Optimization Method 

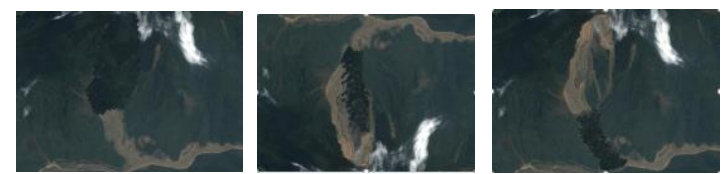

Figure 10. Landslide Simulation Visualization

\section{SYSTEM IMPLEMENTATION AND EXPERIMENTAL ANALYSIS}

We chose the flood as typical mountain disaster and selected three types of devices as terminals for experiments, the experimental terminals and its hardware configuration is shown in Table. 2. In the experiment, laptop was respectively connected to $4 \mathrm{M}$ bandwidth wired network and WIFI network, tablet and smartphone were connected to a $4 \mathrm{M}$ bandwidth WIFI network for experiments. According to performance and screen size of terminal, the rendering frame rate threshold of the laptop is 40 FPS, tablet is 25FPS, smartphone is 25 FPS.

\begin{tabular}{|c|c|c|c|}
\hline Terminals & CPU & GPU & RAM \\
\hline Laptop & $\begin{array}{c}\text { Intel Core } \\
\mathrm{i} 7 / 2.6 \mathrm{GHZ}\end{array}$ & $\begin{array}{c}\text { NVIDA } \\
\text { Geforce960M }\end{array}$ & $16 \mathrm{~GB}$ \\
\hline Tablet & $\begin{array}{c}\mathrm{iPad} \\
\mathrm{A} / 1.5 \mathrm{GHZ}\end{array}$ & $\begin{array}{c}\text { Power VR } \\
\text { SGX544 }\end{array}$ & $2 \mathrm{~GB}$ \\
\hline Smartphone & $\begin{array}{c}\text { iPhone } \\
\text { A9/1.8GHZ }\end{array}$ & $\begin{array}{c}\text { Power } \\
\text { VR GT7600 }\end{array}$ & $2 \mathrm{~GB}$ \\
\hline
\end{tabular}

Table 2. Hardware Configuration of Terminals

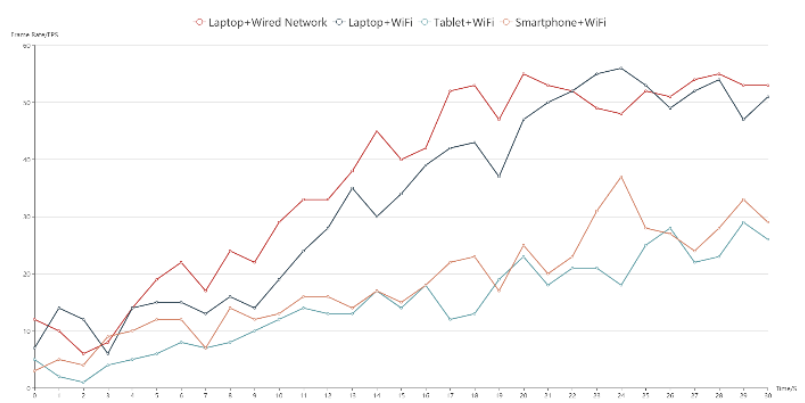

Figure 11. Real-time Rendering Frame Rate of Adaptive Visualization

In the experiment, keeping the viewpoint position, the rendering frame rate during the experiment process is shown in Figure 11. The flood disaster scene visualization on diverse terminals is respectively shown as Figure 12 and Figure 13. From these figures, we can see the rendering frame rate on the laptop was more than 40FPS, the rendering frame rate on the tablet and smartphone was more than 25FPS.
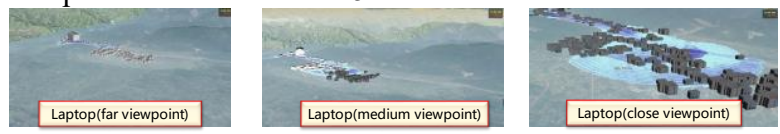

Figure 12. Flood Disaster Visualization on Laptop
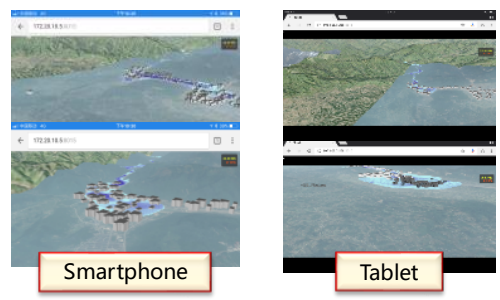

Figure 13. Flood Disaster Visualization on Mobile Device
We also developed a mobile VR application, and designed multiple gaze-based scene interaction mode, users can effectively explore and control 3D flood disaster scenes in VR mode. Flood disaster visualization in VR mode is shown as Figure 14.
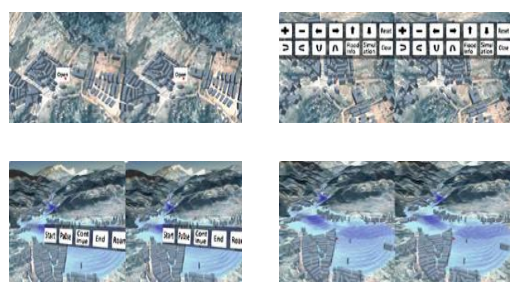

Figure 14. Flood Disaster Visualization in VR Mode

\section{CONCLUSIONS}

We focused on the requirements of the adaptive visualization of complicated mountain disaster scene for diverse terminals. We designed an adaptive visualization of complicated disaster scenes based on $\mathrm{B} / \mathrm{S}$ architecture for diverse terminals, and researched the optimized organization of typical disaster scenes data. In order to improve the efficiency of 3D data traversal and transmission, the loaded data are reduced and the visualization efficiency is improved. According to the terminal performance, network environment, real-time rendering frame rate and the viewpoint information and the diverse optimized organization of various types data in the disaster scenes, a corresponding dynamic scheduling method is proposed to realize the efficient visualization of the complicated disaster 3D scene for diverse terminals. Finally, we select the flood as typical mountain disaster and selected three types of devices as visualization terminals for experiments, and it is demonstrated that the proposed method could meet the requirements of the adaptive visualization of the complicated mountain disaster scenes.

\section{ACKNOWLEDGEMENTS}

This work was supported by the National Key R\&D Program of China(No.2016YFC0803105).

\section{REFERENCES}

Chen, M., Lin, H., Kolditz, O., Chen, C., 2015. Developing dynamic virtual geographic environments (VGEs) for geographic research. In: Environmental Earth Sciences, Vol. 74(10), pp. 6975-6980.

Cui, P., 2014. Progress and prospects in research on mountain hazards in China. In: Progress in Geography, Vol.33(2), pp. 145152.

Denolle, M. A., Dunham, E. M., Prieto, G. A., Beroza, G. C., 2014. Strong ground motion prediction using virtual earthquakes. In: Science, Vol. 343(6169), pp. 399-403.

Hu, Y., Zhu, J., Li, W. L., Zhang, Y. H., Zhu, Q., Qi, H., Zhang, H. X., Cao, Z. Y., Yang, W. J., Zhang, P. C., 2018. Construction and Optimization of Three-Dimensional Disaster Scenes within Mobile Virtual Reality. In: ISPRS International Journal of GeoInformation, Vol. 7(6), pp. 215.

Kim, J. C., Jung, H., Kim, S., Chung, K., 2016. Slope based intelligent $3 \mathrm{~d}$ disaster simulation using physics engine. In: Wireless Personal Communications, Vol. 86(1), pp. 183-199.

Lin, H., Chen, M., Lu, G., Zhu, Q., Gong, J., You, X., Wen, Y. 
N., Xu, B. L., Hu, M. Y., 2013. Virtual geographic environments (VGEs): a new generation of geographic analysis tool. In: EarthScience Reviews, Vol. 126(11), pp. 74-84.

Lin, H., Zhang, C. X., Chen, M., Zheng, X. Q., 2016. On virtual geographic environments for geographic knowledge representation and sharing. In: Journal of Remote Sensing, Vol. 20(5), pp. 1290-1298

Liu, M., Zhu, J., Zhu, Q., Qi, H., Yin, L., Zhang, X., Feng, B., He, H. G., Yang, W. J., Chen, L. Y., 2017. Optimization of simulation and visualization analysis of dam-failure flood disaster for diverse computing systems. In: International Journal of Geographical Information Science, Vol. 31(9), pp. 116.

Yin, L., Zhu, J., Li, Y., Zeng, C., Zhu, Q., Qi, H., Liu, M. W., Li, W. L., Cao, Z. Y., Yang W. J., Zhang, P. C., Cao, Z. Y., 2017. A virtual geographic environment for debris flow risk analysis in residential areas. In: ISPRS International Journal of GeoInformation, Vol. 6(11), pp. 377.

Zhang, Y. H., Zhu, Q., Zhu, J., Liu, M. W., He, H. G., Yang, W. J., Zhang, P. C., 2017. Lightweight Web Visualization of Massive DSM Data. In: Journal of Geomatics Science and Technology, Vol. 34(06), pp. 649-653.

Zhu, Q., Chen, X. W., Ding Y. L., Liu, M. W., He, H. G., Yang, W. J., Chen, L. Y., Cao, Z. Y., 2017. Organization and Scheduling Method of 3D Urban Scene Data Driven by Visual Perception. In: Journal of Southwest Jiaotong University, Vol. 52(05), pp. 869-876. 